\title{
La mémoire de la France Antarctique
}

Frank LESTRINGANT*

Résumé: Les fortunes de la France Antarctique du Brésil sont sans commune mesure avec la brièveté d'une expérience coloniale d'un lustre à peine, de novembre 1555 à mars 1560, restreinte de surcroît à un îlot et à l'immédiate proximité du littoral de la baie de Rio de Janeiro. Je partirai du jugement de l'abbé Prévost, l'auteur de l'immortelle Manon Lescaut, mais aussi de l'Histoire générale des voyages pour retracer la mémoire de la France Antarctique dans l'historiographie française.

Mots-clés: France Antarctique; Mémoire; Prévost.

Les fortunes de la France Antarctique du Brésil sont sans commune mesure avec la brièveté d'une expérience coloniale d'un lustre à peine, de novembre 1555 à mars 1560, restreinte de surcroît à un îlot et à l'immédiate proximité du littoral de la baie de Rio de Janeiro.

Je partirai du jugement de l'abbé Prévost, l'auteur de l'immortelle Manon Lescaut, mais aussi de l'Histoire générale des voyages. Dans ce dernier ouvrage, Prévost recopie d'après Jean de Léry le "Colloque de l'auteur et d'un sauvage, montrant qu'ils ne sont si lourdauds qu'on les estimait"1, passage que reprend l'abbé Raynal dans l'Histoire des deux Indes et qu'il commente ainsi :

L'unique monument précieux de leurs courses infructueuses, est un dialogue qui peint d'autant mieux le bon sens naturel des sauvages, qu'il est écrit dans ce style naïf qui

\footnotetext{
* Centre de recherche sur la création littéraire en France à la Renaissance - Université de Paris IV-Sorbonne - 75230 - Paris França. E-mail: frank.lestringant@wanadoo.fr
} 
caractérisait il y a deux siècles, la langue Française, et où l'on retrouve des grâces qu'elle doit regretter ${ }^{1}$.

Afonso Arinos de Melo Franco l'a exprimé dans un raccourci saisissant : O Indio brasileiro e a Revoluçâo francesa ${ }^{2}$. Il y a bien un lien entre l'expérience de la France Antarctique et la Révolution française, un lien indirect et détourné, qui passe par Léry, Montaigne, Prévost et Raynal, mais un lien profond et essentiel. Il faut ici rappeler la phrase célèbre de Jean de Léry qui pourrait servir de conclusion à son Histoire publiée à Genève en 1578 : "Je regrette souvent que je ne suis parmi les sauvages" ${ }^{\prime \prime}$. Et il ajoute : "... auxquels j'ai connu plus de rondeur qu'en plusieurs de par-deçà, lesquels à leur condamnation, portent titre de Chrétiens" ${ }^{\prime \prime}$. Deux ans plus tard, Montaigne lui fait écho dans "Des Cannibales": "Chacun appelle barbarie ce qui n'est pas de son usage"

Mais ce n'est pas la question anthropologique ni celle du relativisme culturel que je voudrais agiter ici, et dont il a été suffisamment traité ailleurs. Sans doute, de ce point de vue, l'échec de la France Antarctique a été particulièrement fécond. C'est l'échec colonial, en effet, qui a été au point de départ de l'ébranlement des certitudes qui va conduire, après bien des détours et des vicissitudes, à la reconnaissance de l'égalité des hommes et des cultures. Sans l'échec de 1560, Montaigne n'aurait sans doute pas écrit "Des Cannibales" comme il l'a écrit, ni Jean-Jacques Rousseau le second Discours, Des fondements et de l'origine de l'inégalité parmi les hommes.

\section{La mémoire de la France Antarctique dans l'historiographie en France (XVIe-XVIIIe siècles)}

Laissant de côté cette perspective anthropologique que je viens de retracer à grands traits, je voudrais m'intéresser de plus près à la mémoire de la France Antarctique dans l'historiographie française, en descendant ce fil jusqu'aux toutes 
récentes dérives romanesques $d u$ tournant $d u$ troisième millénaire. Cette mémoire de la France Antarctique, en France du moins, est une mémoire infidèle et sporadique, qui se condense en des moments clés du débat politique et religieux, à la veille de la signature de l'édit de Nantes, plus tard au lendemain de l'assassinat d'Henri IV et sous la minorité de Louis XIII, plus tard encore autour de la Révocation, enfin durant les décennies qui préparent la Révolution, aujourd'hui à nouveau, au temps de la globalisation triomphante. Cette histoire de la France Antarctique est faite de plus d'oublis que de mémoire, de plus de légendes que de réalités. Elle nous intéresse toutefois, bien qu'elle paraisse souvent négliger ce qui, à nos yeux, constitue l'essentiel, la découverte d'un Nouveau Monde et d'une nouvelle humanité, pour privilégier au contraire des aspects anecdotiques, voire pittoresques ou incongrus, qui ne soulèvent plus aujourd'hui que l'hilarité ou le dédain sceptique. Ces aspects que nous jugerions accessoires, tels que la controverse eucharistique resurgie sous les tropiques, la question de la sincérité de Villegagnon et de ses convictions intimes ou encore la légitimité de la révolte face au tyran, furent pourtant essentiels sur le moment et motivèrent des engagements contradictoires d'où résulta la catastrophe tragique qui conclut l'épisode colonial.

\section{Portrait de Villegagnon en " moyenneur "}

Pour bien comprendre les errements apparents de cette mémoire française diluée sur quatre siècles, il faut donc en revenir aux enjeux fondamentaux qui furent ceux de la colonie de 1555-1560.

Il s'agit, bien sûr, d'une entreprise destinée à pérenniser la présence française au Nouveau Monde en battant en brèche le monopole ibérique sur ce continent. Le choix du Brésil s'expliquait par les relations de traite déjà anciennes avec les tribus Tupinamba de la côte et la présence nombreuse de truchements normands ou saintongeais parmi les Indiens. Ce 
choix était justifié aussi par la moindre résistance que le Portugal était censé opposer aux ambitions de conquête du roi de France. Un lustre plus tard à peine, quand l'amiral de Coligny décidera d'implanter une nouvelle colonie française sur les côtes de la Floride, il prendra de plus grands risques en s'attaquant directement à l'Espagne et au coeur de l'empire colonial de celle$\mathrm{ci}$, à savoir la carrera de las Indias que l'établissement menaçait directement. La riposte fut foudroyante et l'entreprise, plus éphémère encore que celle de la France Antarctique et assurément plus sanglante, prit fin au bout de trois ans (15621565).

Ces deux épisodes, qui se situent dans l'étroite continuité l'un de l'autre, offrent bien des similitudes. Ils mettent en oeuvre des stratégies très voisines, tout en accentuant la prise de risque dans le second cas. À l'occupation du territoire se conjugue en effet le contrôle d'une voie maritime. Charlesfort et surtout Fort Caroline, établi plus au sud en Floride, menaçaient la route des galions espagnols. Fort Coligny, à l'entrée de la baie de Guanabara, constituait une menace tout aussi réelle, l'amiral Guedes l'a rappelé hier, pour la route portugaise des Indes ou celle du détroit de Magellan.

Mais il y avait aussi d'autres enjeux, sur lesquels je voudrais insister à présent. La France d'Henri II, certes prospère par rapport à la période des guerres de Religion qui suit, est déjà une France en crise. La Réforme a fait d'immenses progrès, propagée d'abord depuis l'Allemagne luthérienne et les cantons suisses, se développant ensuite de façon autonome, même si elle commence à être structurée de l'extérieur, depuis Genève, par le Français Jean Calvin exilé à partir de l'affaire des Placards contre la Messe (octobre 1534).

Après la fin des guerres d'Italie, Henri II a établi comme priorité la lutte contre l'hérésie, et les débuts de son règne (15471559) correspondent à la recrudescence des "Feux", c'est-à-dire des bûchers où l'on condamne les protestants. Mais cette politique des "bûchers du roi", pour reprendre la formule de David El-Kenz ${ }^{6}$, aboutit à un échec, d'autant que l'entourage même du roi est divisé. Le duc de Guise est le plus fervent 
défenseur du catholicisme, mais l'amiral de Coligny qui patronne l'expédition de Villegagnon glisse peu à peu vers la Réforme à laquelle il adhère en 1558-1559 lors de sa captivité consécutive au siège de Saint-Quentin.

En réalité, une troisième solution paraît se dessiner, intermédiaire entre la fidélité envers l'Église de Rome et la rupture brutale avec celle-ci. "Ni Rome ni Genève", pour reprendre le titre du beau livre de Thierry Wanegffelen ${ }^{7}$, tel pourrait être le slogan de ce tiers-parti, auquel, de toute évidence, appartient Villegagnon, du moins à l'origine et avant les événements de $1557^{\circ}$. Ce tiers parti, c'est celui des "moyenneurs", partisans, sous l'autorité du roi de France, d'une Réforme intérieure de l'Église qui conduirait à desserrer les liens avec Rome sans embrasser pour autant la Réforme calviniste. Ce serait en quelque sorte une solution gallicane à la crise religieuse, l'équivalent exact de ce que fut l'anglicanisme promu par Henri VIII d'Angleterre, puis pérennisé par sa fille Élisabeth, après la parenthèse catholique de Marie Tudor. Villegagnon, cela est incontestable, fait partie de ces moyenneurs gallicans qui cherchent à refonder l'Église française sur des bases propres, nationales et monarchiques, dans une double fidélité à l'Évangile et à la personne du roi. Un roi qui est assimilé au Christ et dont la sacralité est réaffirmée avec d'autant plus de vigueur.

Avec Villegagnon, l'idéal gallican des moyenneurs va rejoindre l'utopie brésilienne. En effet, si l'on peut parler d'une utopie de la France Antarctique ${ }^{9}$, c'est d'abord en ce sens précis, tout à la fois religieux et politique, d'une refondation chrétienne ou, comme Calvin le disait dans sa somme théologique, d'une "institution chrétienne" en terre étrangère. Cette idée se dégage des nombreux traités de controverse que Villegagnon a composés après son retour en France pour répondre aux accusations de trahison dont il était l'objet de la part des protestants. Villegagnon, sans nul doute très sincèrement, a cherché à refonder la communauté chrétienne sur des bases, non pas nouvelles, car c'est tout le contraire d'un révolutionnaire, mais sur les fondements retrouvés et raffermis de la religion chrétienne, c'est-à-dire sur l'Écriture sainte et la tradition des 
Pères. Cet effort de retour aux sources est parallèle à celui de la Réforme, mais il s'en distingue par le souci prioritaire qu'il a de la concorde, c'est-à-dire de l'unité de tous les chrétiens. La distinction est d'importance. Si Villegagnon, en bon moyenneur, est un adepte de la concorde, cela veut dire qu'il recherche avant tout la réunion de tous les chrétiens sur un même dogme; en aucun cas, ce n'est un adepte de la tolérance - et la suite des événements va cruellement le prouver.

En définitive, Villegagnon, qui est théologien autant qu'homme de guerre et de conquête, va mettre à profit l'occasion que lui donne l'entreprise de la France Antarctique pour tenter cette expérience d'une société chrétienne "réformée" ou restituée. Les circonstances sont d'autant plus favorables que la place est vide. Le Brésil de 1555 offre la table rase idéale pour fonder sur nouveaux frais une Église que n'obère pas, d'emblée, un long passé de décadence et de compromissions. C'est le lieu rêvé pour revivre les temps bénis de l'Église primitive.

Il s'agira donc de restituer le corps mystique de l'Église et le corps mystique de l'État, ce qui pour Villegagnon est tout un, de le restituer en connaissance de cause et après discussion préalable. Cela explique qu'au départ Villegagnon n'interdit pas la controverse, mais qu'au contraire il la suscite. Sitôt débarqués les quatorze "Genevois" au printemps 1557, il entreprend la discussion théologique avec les deux pasteurs Richer et Chartier. Très vite la dispute s'envenime au sujet de l'Eucharistie, les protestants chapitrés par Calvin avant leur départ de Genève refusant la transsubstantiation aussi bien que la consubstantiation luthérienne, cette dernière ayant quant à elle les faveurs du dissident Jean Cointa. L'affaire se termine tragiquement, début 1558, par l'exécution de trois des quatorze huguenots, après un simulacre de procès. Mais l'important est au départ la démarche de Villegagnon : il préconise la "conférence", c'est-à-dire le débat, de manière à dégager une vérité acceptable par tous. C'est cette même démarche qui sera reprise, cette fois à l'échelle du royaume, par Catherine de Médicis et le chancelier Michel de L'Hospital à la veille des guerres de Religion. Tel sera l'objet du colloque de Poissy réuni 
aux mois d'août et de septembre 1561. Et ce sera là aussi un échec, pour des raisons identiques.

Villegagnon, comme plus tard L'Hospital, engage la dispute dans le but d'établir la concorde entre chrétiens. C'est le contraire qui en résulte dans les deux cas, de sorte que la France Antarctique répète par avance, et en miniature, le drame des guerres de Religion. Le drame qui se joue sur le théâtre du Brésil fait donc figure de répétition générale. Il laisse espérer une solution qui, pour une majorité de clercs et de politiques, paraît alors la plus viable et la seule acceptable. Et presque aussitôt il enregistre l'échec de cette via media. Mais de la démonstration de cet échec nul n'a voulu tenir compte, car chacun dès lors a campé sur ses positions. Les protestants, bien sûr, mais aussi Villegagnon qui, après avoir été séduit par le rôle d'arbitre, s'est rangé du côté de l'orthodoxie catholique. Sans nul doute pour se blanchir aux yeux de la Cour et se dégager de toute compromission avec un parti dont il ne connaissait pas l'intransigeance. À la tentative sincère de recherche d'une solution commune succède par conséquent, courant 1557, le différend où chacun s'efforce d'imposer ses vues, y compris par la violence et la coercition.

Les enjeux tout à la fois théologiques et politiques que met en lumière l'expérience avortée de la concorde en France Antarctique sont cruciaux. On peut les résumer ainsi :

1) La définition de la communauté chrétienne, que menace la crise eucharistique.

2) La relation entre le corps du roi et le corps de l'Église, ou, pour le dire en termes plus modernes, les rapports de l'Église et de l'État.

Questions corollaires :

3) L'existence de plusieurs confessions à l'intérieur d'un même État, cette coexistence étant longtemps considérée comme impensable, car fautrice, pensait-on, de désordre et de troubles.

4) Le droit ou le devoir de révolte contre la tyrannie, cette dernière, au Brésil, étant incarnée par Villegagnon, une fois qu'il 
s'est converti à la manière forte. Cette question de la résistance légitime au tyran est à mettre en rapport avec l'édition et la diffusion par les protestants français, dès les lendemains de la Saint-Barthélemy, du Discours de la Servitude volontaire d'Étienne de La Boétie.

5) L'autonomisation de la raison politique ${ }^{10}$ et la question de la tolérance.

On s'aperçoit que ces questions sont en fait très modernes, dès lors qu'on les dégage de la gangue scolastique qui les enrobe et qui a permis aux hommes de la Renaissance de les formuler une première fois ; dès lors aussi que l'on dépasse les querelles de personnes pour aller à l'essentiel,

La controverse théologique au Brésil (1557)

L'originalité de l'expérience conduite en France Antarctique tient en particulier au fait que la question religieuse fut l'une des causes premières de l'échec colonial. La controverse eucharistique qui connut un épilogue tragique en janvier 1558, annonce la grande cassure qui, à l'issue du colloque de Poissy, en septembre 1561, prélude aux guerres de Religion. Aux tenants de la Présence réelle et corporelle du Christ s'opposent les partisans d'une conception purement symbolique du sacrement, prêts à remplacer, le cas échéant, le vin par la bière de mil et le pain par la farine de manioc, les nourritures les plus communes entre les sauvages du Brésil. Querelle byzantine, dira-t-on, et pour le moins incongrue, en une région du monde où elle eut pour toile de fond la forêt tropicale et pour témoins d'authentiques cannibales, dont on imagine après coup la surprise et l'incrédulité ${ }^{11}$.

La dispute posait en fait une question anthropologique essentielle et revenait à s'interroger sur les fondements mêmes $\mathrm{du}$ christianisme: comment la "vraie" religion avait-elle pu triompher de la malédiction originelle, cette contrainte du sacrifice généralement observée à travers le monde, en 
particulier chez les peuples les plus éloignés de nos contrées? De quelle manière surtout elle paraissait près d'y retomber à tout moment, en dépit de l'Évangile de paix et d'amour qu'elle avait le devoir d'annoncer à la terre?

Tout part, du côté protestant, d'une sorte de télescopage entre la découverte de l'Indien et la sainte horreur que suscite le frère ennemi catholique, "mange-Dieu" plutôt que chrétien, théophage mais aussi, et nécessairement, "théochèze"12. Le catholique, en effet, rencontre le cannibale et se révèle bien pire. Le rite cannibale partage le caractère communautaire avec le sacrifice de la Messe, qui réalise la communion des fidèles dans la consommation du Fils de Dieu, présent réellement et en substance dans l'hostie consacrée.

Mis à part cette dimension sociale et universelle du rite, tout sépare les deux cuisines. Le festin tupinamba clôt un processus culinaire normal allant du vivant au mort et du cru au cuit. Il tend à réduire, comme c'est le but de toute cuisine cannibale, le scandale de l'autophagie. Manger du même, voilà qui répugne aux Brésiliens aussi bien qu'aux chrétiens réformés de France et de Navarre. L'horreur suscitée par le "boucan" - ou gril de bois dur - chargé de membres humains tient précisément à la ressemblance de la viande morte avec la chair vivante, dans ses parties les moins comestibles: tête, mains, pieds, qui sont aussi les moins transformables par la cuisson. De plus, cette préparation alimentaire va de l'unité anatomique de l"homme sur pied" à la diversité des plats fumés ou rôtis, liquides ou solides, qui sont distribués aux convives selon l'âge, le sexe et le rang social de chacun: graisse pour les vieilles qui en sont particulièrement friandes, sang dont on barbouille les enfants à la mamelle, chair musculeuse pour les hommes adultes, parties génitales pour les femmes enceintes, etc.

L'Eucharistie catholique procède exactement au rebours, et renverse par là même le processus culinaire normal. Elle transforme le cuit initial (le signifiant pain) en un cru symbolique (la chair du Christ vivant). Au lieu d'opérer le découpage de l'aliment non préparé et de séparer ses différents constituants, elle réalise la fusion d'une dualité (vin et sang, liquide distinct du 
solide) en une seule entité (l'hostie vivante reproduisant le corps glorieux du Christ).

À travers cette critique de l'Eucharistie par le cannibalisme des Tupinamba, les calvinistes entendent montrer que l'hérésie" catholique de la transsubstantiation est double : d'abord, parce qu'elle se fonde sur la perversion anthropophage; ensuite, parce qu'elle inverse cette anthropophagie elle-même, en en faisant une opération régressive de retour au cru.

\section{Les Tupinamba de l'édit de Nantes (1597)}

Les Tupinamba du Brésil reprendront du service beaucoup plus tard dans la polémique huguenote. Lorsque, à la veille de la conclusion de l'édit de Nantes, les protestants s'inquiètent des exhumations auxquelles procèdent un peu partout les catholiques, vidant leurs cimetières de tout cadavre "hérétique", ils ne peuvent faire moins que d'invoquer l'exemple des "Toupinambaux" (les Tupinamba) et des "Margajas" (les Maracaia), moins barbares en comparaison. C'est ainsi que s'exprime au printemps 1597 le libelle anonyme des Plaintes des Eglises Réformées de France:

Les Margajas, les Toupinambaux remplissent leurs entrailles de la chair de ceux qu'ils ont mis à mort. Avec détestation, nous les en appelons barbares, Sauvages. Et toutefois, cette cruauté n'est point de Margajas à Margajas : de Toupinambaud à Toupinambaud. Le Toupinambaud ne mange que le Margajas, et le Margajas n'est glouton que du Toupinambaud. Encore ne le font-ils que pour rendre la pareille ; que pour se venger de ceux qui en bravade, quoique ayant la mort entre les dents, leur disent, j'ai mangé ton frère : et mangerais tes enfants si j'avais plus de vie. Pour le faire, ne violent point les tombeaux ${ }^{13}$.

On reconnaît le procédé de la "révolution sociologique" mis au point par Montaigne dans "Des Cannibales" et par Léry dans l'Histoire d'un voyage, repris et systématisé plus tard par Montesquieu dans Les Lettres persanes, et qui consiste à 
intervertir les regards de l'observateur et de l'observé, le point de vue de l'Européen et celui du prétendu sauvage ${ }^{14}$. Il en ressort que le plus barbare n'est pas celui qu'on pense. On constate également le succès de l'adage venu de Plaute, Homo homini lupus ${ }^{15}$,transposé ici sur le mode cannibale et acclimaté à l'anthroponymie brésilienne. On voit enfin par cette référence à la haine légendaire des "Toupinambaux" et des "Margajas" (ou Marakaia) que Jean de Léry est devenu à cette date une sorte de "classique du protestantisme". Une gravure de la deuxième édition de l'Histoire d'un voyage montrait le "combat entre les sauvages Toüoupinambaoults et Margajas Ameriquains."16, une féroce empoignade où les Indiens nus et emplumés se battaient à coups de massues et de flèches, mais aussi d'ongles et de dents.

Une fois encore, le catholique est plus inhumain que le Cannibale qui donne son ventre pour sépulture à l'ennemi et fait sienne sa chair. Une nouvelle fois, cette barbarie catholique retourne en quelque sorte la barbarie exotique : au lieu de digérer l'intrus fibre après fibre, elle l'expulse violemment, jusqu'à arracher à la terre "une chair pourrie, puante, un crâne hideux, des os tout démanchés, et vermoulus, tout pleins d'horreur"117. Le libelle de 1597 interroge : "Mais quel nouveau goût, quelle bonne odeur avez-vous trouvée ès corps morts, et corps pourris, et pourris depuis dix ans ?" Comment expliquer cet acharnement autrement que par un appétit insatiable et dénaturé ? "Et ne vous chaut quoi, pourvu que repaissiez cette furieuse passion qui ne s'assouvit jamais". Une passion de haine et de vengeance, celle-là même qui anime les sauvages les uns contre les autres, mais on l'a vu, seulement entre ennemis héréditaires, et selon un code qui exclut la profanation des sépultures.

On se trouve ici, en termes d'anthropologie structurale, aux antipodes de la cuisine cannibale. Le catholique exhumateur de cadavres protestants vieux de dix ans se repaît symboliquement tout au moins - de pourriture, d'une viande corrompue et immangeable, qui, loin d'être passée par le processus civilisateur du feu de cuisine, a été abandonnée aux 
facteurs naturels de la décomposition. Le catholique persécuteur, et le président Florimond de Raemond le tout premier, qui avait fait prononcer par le parlement de Bordeaux "que tous les corps de ceux de la Religion qui depuis dix ans auraient eu terre en quelque Cimetière, seraient arrachés de leurs tombeaux ${ }^{118}$, se situe, non du côté de la culture comme le cannibale du Brésil, mais du côté d'une nature dont il raffole sous les espèces les plus répugnantes et les plus bestiales.

De sorte que le catholique, qui tout à la fois affirme la réalité de la transsubstantiation et déterre le corps mort de son ennemi pour le jeter aux chiens et aux loups, conjugue anthropophagie et anthropémie ${ }^{19}$. Il mange le sacro-saint cadavre du Christ et vomit, presque littéralement, celui du protestant, additionnant les infamies en sens contraires.

Dans le désespoir qui l'anime, le pamphlet protestant jette la malédiction sur toute forme de cimetières, tout en réaffirmant la foi dans la résurrection au jour du Jugement :

$\mathrm{Au}$ reste que nous peut-il meshui servir de bien clore les Cimetières, de soigneusement couvrir les fosses? On le faisait pour empêcher que les bêtes ne violassent ces lieux et naturellement sacrés, et inviolables pour l'honnêteté? O Dieu! si que se trouve horrible aux bêtes, est aujourd'hui permis aux Français ! Et que nous doit-il chaloir qu'un pourceau fouillant du groin nous découvre ; ou qu'un Français fouissant nous déterre ? Lequel nous vaudra donc mieux, qu'un Loup dévore notre charogne, ou que nos citoyens en repaissent leurs yeux en contentant leur rage? Certes ni l'un ni l'autre n'empêchera, qu'en ces mêmes os, en ceste même chair nous ne voyions notre Rédempteur, qui approche, et rendra selon sa justice, oppression à ceux qui nous oppressent, et relâche à nous qui sommes oppressés lorsqu'il apparaîtra du Ciel avec les Anges de sa puissance $^{20}$.

On ne saurait se méprendre à la lecture d'une telle page. Le calvinisme, il est vrai, professait une indifférence de principe quant au devenir de la dépouille mortelle. Calvin lui-même ordonna que son cadavre fût enterré à la manière commune, sans signe distinctif sur son tombeau. Ce n'est pas toutefois que, 
selon une erreur d'interprétation souvent répétée, il demanda à être jeté à la fosse commune ${ }^{21}$. Peu importe, en théorie, que la chair insensible pourrisse lentement au fil des hivers, ou qu'elle soit dévorée par les porcs, les loups ou même les hommes. Ce devenir charnel n'engage nullement le destin de l'âme après la mort : il y aurait là une superstition des plus grossières. Mais dès lors que cette indifférence vis-à-vis du cadavre est ressentie comme l'effet d'une contrainte, dès lors qu'elle est dictée en quelque sorte par l'intolérance de l'adversaire, cette indifférence forcée devient insupportable, aussi scandaleuse, en vérité, que l'attachement trop scrupuleux au corps.

Pourquoi s'en prendre ainsi au corps des huguenots ? À cette question Bernard Cottret apporte une réponse très plausible: "Sans doute parce que les premiers ils ont désacralisé l'au-delà des catholiques"22. Dès le début des troubles de Religion, ils ont brisé les tombes, violé la sépulture des saints et des rois, tourné en ridicule le Purgatoire et même l'Enfer. Par conséquent, les catholiques se vengent sur leurs cadavres. Mais voici que "l'épuration" des cimetières provoque un choc en retour inattendu. Nonobstant le mépris qu'ils affichent d'ordinaire pour l'enveloppe charnelle, les réformés s'insurgent contre une profanation qui les touche à présent directement.

On mesure à ce point la persistance d'une sourde hantise en filigrane de la croyance officielle. Le refus d'un culte exaltant la chair du Christ et la haine de l'idolâtrie trahissent, jusque dans leur expression la plus forcenée, une bien étrange fascination. La contradiction inscrite dans la "sainte horreur" huguenote est intime et profonde ${ }^{23}$. Le haut-le-cœur que ressentent les protestants de 1597 est d'autant plus violent, leur réaction d'autant plus viscérale qu'il s'agit cette fois de leur propre chair, non de la chair d'autrui, non de celle d'un Christ lointain remonté au ciel depuis le temps des apôtres.

À l'horizon de cette douleur modulée dans les règles de l'art, exprimée selon les lois du genre, exacerbée sur le mode pathétique et oratoire de la remontrance au roi, se dessine l'échéance ultime, le temps eschatologique du Jugement dernier. C'est, plutôt qu'une crainte, une attente frémissante et un espoir 
vertigineux. Seul le retour du Christ sur la terre permettra de rétablir les justes dans leurs droits et de punir les méchants comme ils le méritent. Seul surtout il mettra fin à la malédiction et à la souillure irrémédiablement attachées ici-bas à toute chair périssable. Mais sans attendre, et de manière plus réaliste ou plus pragmatique, les Plaintes des Eglises sont une mise en demeure adressée au roi Henri IV, naguère protestant et maintenant catholique. En tant qu'image de Dieu sur la terre, c'est à lui qu'il appartient, selon ses prérogatives, d'exercer la justice. Faute de quoi, l'homme qu'il est aussi et qu'il n'a pas cessé d'être pourrait bien s'en ressentir au jour de gloire et de colère. La menace n'est pas explicitement formulée, mais elle est sous-jacente à cette péroraison inspirée, emplie d'un souffle prophétique, qui recrute de concert Toupinambaux et Margajas dans le combat pour la paix des cimetières.

Les Martyrs huguenots du Brésil au filtre de l'Anti-Martyrologe (1622)

Le second point de la postérité confessionnelle de la France Antarctique est à peine moins dramatique. Il s'agit de la question du martyre. La brève expérience de concorde religieuse en 1557-1558 s'était soldée à Guanabara par l'exécution de trois protestants, jetés les fers aux pieds dans la baie après qu'ils eurent refusé d'abjurer sous la contrainte. Sans tarder, ces trois martyrs huguenots du Brésil allaient entrer dans l'hagiographie protestante $^{24}$. Dès 1564, l'Histoire des martyrs rassemblée par l'éditeur genevois Jean Crespin accueillait en deux chapitres distincts l'odyssée des trois huguenots, leur confession de foi et leur supplice. Jean du Bordel, Matthieu Vermeil et Pierre Bourdon étaient admis un peu plus tard dans le recueil des Hommes illustres en pieté et doctrine de Théodore de Bèze, le compagnon et successeur de Calvin à la tête de l'Église de Genève ${ }^{25}$.

Cette histoire tragique n'allait pas échapper à la guerre des martyrs que se livraient, par martyrologes interposés, les églises 
protestante et catholique. À ce qu'il appelle le "Pseudomartyrologe de Geneve", Jacques Severt, théologal de Lyon, répliqua en 1622 par un volumineux Anti-martyrologe, ou Verité manifestée contre les Histoires des supposés martyrs de la Religion pretendue reformée, imprimées à Geneve onze fois ${ }^{26}$. Severt contestait l'Histoire des martyrs à la fois sur le fond et sur la forme. Sur le fond, bien évidemment, ces martyrs, s'étant séparés de l'Église seule et véritable, étaient des martyrs du diable, et non pas de Dieu. En toute rigueur, Severt appliquait l'adage, allégué notamment par saint Augustin dans son combat contre les donatistes et repris à date récente par les controversistes des deux camps, le catholique Florimond de Raemond et le protestant Simon Goulart : Martyrem non facit poena, sed causa ; "ce n'est pas la peine - autrement dit la souffrance -, mais la cause qui fait le martyre ${ }^{127}$. Prenons le cas, expliquait Raemond, d'un anabaptiste capturé par un Turc, empalé ou mis en croix pour la défense de ses convictions erronées. Il ne devra pas pour autant être considéré comme un martyr, puisqu'à la vérité chrétienne qu'il confesse, il mêle le mensonge. Son martyre ne vaut pas. Le supplice ne lui profite de rien; bien au contraire, il lui fournit un avant-goût des peines éternelles qu'il s'apprête à souffrir ${ }^{28}$.

Sur la forme, Severt a procédé à une minutieuse enquête dans les parlements de tout le royaume, de manière à réduire, unité par unité, le nombre total des "pseudo-martyrs" réformés. Son enquête relève de ce que l'on appellerait aujourd'hui une démarche révisionniste, voire négationniste au bout du compte. Pour ce qui est du Brésil, Severt s'est proposé, comme il le dit d'entrée de jeu, de "questionner et raccourcir les divorces des nouvelles conquêtes faites au pays de Brésil, partie méridionale de l'Amérique" 29 . Au passage, Severt s'en prend au "collecteur Jean Deléry", c'est-à-dire Jean de Léry, "calcographeur aussi des faux Martyrologes ${ }^{1130}$, pour dénoncer le caractère partial de son témoignage. Severt reproche à Léry de faire de Villegagnon un calviniste repenti, autrement dit un hérétique.

Certes Calvin, qui l'avait connu à la faculté de droit d'Orléans, le pasteur Pierre Richer ou Jean de Léry avaient cru 
un moment trouver en Villegagnon un "second saint Paul"13, un persécuteur qui, par l'effet d'une grâce soudaine, s'était engagé corps et âme au service des persécutés. Illusion de courte durée: très vite le chevalier de Malte, qui ne s'était rallié qu'en apparence et peut-être par méprise à la Réforme de Calvin ${ }^{32}$, se rétractait et retrouvait son rôle de toujours, le rôle d'un oppresseur méritant par la condamnation et la mise à mort des trois martyrs huguenots le surnom de "Caïn de l'Amérique"33.

Severt retient donc la thèse, qui est aussi celle des protestants, d'un Villegagnon simulateur, mais d'un simulateur à bon escient : "Il y feignit l'autre parti à bonne intention", et de surcroît brièvement, "environ une semaine ou deux", à seule fin de démasquer l'adversaire et de constater jusqu'où la malignité de ce dernier pouvait s'étendre. Severt approuve la sentence de mort prononcée et exécutée par Villegagnon à l'encontre des trois artisans huguenots revenus vers lui. Leur hétérodoxie est suffisamment démontrée par les dix-sept articles de leur confession de foi, que l'auteur de l'Anti-martyrologe épluche un à un. L'Eucharistie en particulier est "crayonnée" par eux "d'un pinceau noirci à la Calvinienne ${ }^{1134}$. En définitive, on ne saurait parler de cruauté à leur égard, mais d'une "juste exécution".

Severt insiste pour finir sur le contraste résultant de l'échec protestant et de la réussite missionnaire catholique. Il oppose de ce point de vue la faillite de la France Antarctique et le succès de la France Equinoxiale de Razilly, la Ravardière et Pézieu dans lîle de Maranhâo, ignorant que cette entreprise, elle aussi financée et conduite à l'origine par des protestants, n'avait pas été plus heureuse que l'autre. Il s'étend à loisir sur le bilan négatif de l'entreprise huguenote. Des rêves d'empire de Coligny au Nouveau Monde non plus que des espérances missionnaires de Calvin au Brésil, il ne reste rien, pas la moindre trace. Pure imposture que ce grand nom de France Antarctique couvrant des zones entières de la mappemonde, emplissant les cartes géographiques universelles: "Tant y a, qu'aujourd'hui on n'y sait aucun Français depuis lors demeuré résident" ${ }^{135}$. Le Brésil français est donc passé aux profits et pertes, à l'exception de la brève enclave de Saint-Louis du Maranhâo, terre de mission des 
capucins Claude d'Abbeville et Yves d'Évreux sous la régence de Marie de Médicis.

Il est facile, en revanche, d'opposer à "cette vanterie Genevesque de sa secte qu'elle prétend loin répandue", la réalité de l'expansion catholique en Orient et en Occident. Il s'agit, en premier lieu, du Japon, vitrine de la mission jésuite au tournant du XVIIe siècle, avant le brutal retour de fortune sur le front missionnaire : "Car d'un côté nous lisons le Japon pieçà reconnu obéissant au Saint-Siège [...], île grande comme l'Angleterre et l'Écosse". L'Angleterre et l'Écosse rebelles au Pape trouvent, par l'intervention de la Providence, une sorte de compensation ou de contrepoids dans ces îles nouvellement gagnées aux lumières du catholicisme, à l'Extrême-Orient du monde connu. C'est, en second lieu, la Nouvelle-France d'Amérique qui, dans ces mêmes années, passe d'un simple projet et d'un simple nom sur la carte à un commencement de réalisation. Ainsi donc, sur ces deux bords extrêmes et situés aux antipodes l'un de l'autre, la chrétienté catholique se propage et fait tache d'huile, à la différence de l'hérésie confinée dans quelques régions $d^{\prime}$ Europe $^{36}$. Preuve par l'évidence géographique de la vérité du dogme catholique, apostolique et romain.

On perçoit les enjeux qui sous-tendent un tel débat. Severt est un soldat de la Contre-Réforme, pour lequel l'intérêt national importe moins que la lutte confessionnelle qui transcende la diversité des langues et des peuples dans l'unité religieuse à atteindre. La leçon d'Henri IV, douze ans après le coup de couteau de Ravaillac, n'a pas été entendue. La religion, pour ce zélateur obscur, prime décidément la politique, une religion toujours sur la défensive malgré ses succès récents sur le front des missions lointaines et arc-boutée sur le maintien jaloux de ses dogmes face à une Réforme qu'elle veut croire moribonde.

La France Antarctique et la Révocation de l'édit de Nantes (1685)

À la fin du même siècle, c'est-à-dire près d'un siècle et demi après la chute de Fort Coligny, Pierre Bayle constate dans son Dictionnaire historique et critique que cette entreprise, quand 
bien même lointaine et presque oubliée, "sert souvent d'épisode dans les ouvrages de controverse ${ }^{1137}$. Et de fait, Bayle, en réponse aux catholiques Maimbourg et Bossuet, entend prouver la fausseté de la thèse qui fait des protestants des diviseurs euxmêmes divisés, des fauteurs de troubles et des schismatiques en désaccord les uns avec les autres et réglant leurs différends par l'épée. Une caricature, fameuse depuis Ronsard et la Continuation du Discours des Miseres de ce temps, montre les ministres en manteau de cavalier et l'épée au côté. Ainsi équipés, ils prêchent "une Évangile armée",

Un Christ empistolé tout noirci de fumée,

Portant un morion en tête, et dans la main

Un large coutelas rouge de sang humain ${ }^{38}$.

Ce cliché a la vie dure. Malgré le retour à la paix dès 1598 et l'indéfectible fidélité au roi des sujets protestants sur l'espace de trois générations, c'est toujours l'image du rebelle qui demeure, inlassablement reprise par les controversistes catholiques, en particulier dans les années qui préparent la révocation de l'édit de Nantes. À les en croire, le protestantisme serait intrinsèquement un facteur de division et, qui plus est, un défi au principe d'unité qui préside au gouvernement monarchique, un principe sacrosaint en régime de droit divin.

Or la France Antarctique a servi de laboratoire. En raison même, paradoxalement, de sa brièveté et de sa faible extension dans l'espace, cette expérience d'une cohabitation ratée, parce qu'impossible, apparait exemplaire. C'est le microcosme où s'est jouée de manière transparente, et comme en miniature, une répétition générale du conflit religieux à venir. De plus, cette expérience malheureuse a été suivie d'un déluge de publications de controverse, favorisée par la courte période de tolérance inaugurée par Michel de L'Hospital à la veille des guerres de Religion. De cette manière, les polémistes disposaient d'une panoplie toute prête pour reprendre le combat, le moment venu.

D'où le resurgissement périodique de ce récif jamais totalement englouti du différend religieux au cours de l'âge 
classique et sa nouvelle actualité dans les années 1680, lorsque les partisans de la Révocation s'efforcent de démontrer par différents précédents historiques, la dissidence naturelle, obligatoire et constitutive du protestantisme français.

La grande voix de Pierre Bayle répondra à cet acte d'accusation dressé simultanément par le père Maimbourg, Varillas et Bossuet, en établissant que la rébellion des huguenots du Brésil est une forgerie de Villegagnon, répétée par Jacques Severt et ses suiveurs. Si les protestants, du reste, l'avaient voulu, ils auraient eu facilement raison de Villegagnon, dont les forces sur place étaient inférieures, comme l'atteste Jean de Léry ${ }^{39}$. Les calvinistes de Guanabara ont préféré à la révolte ouverte, qui était à portée de main, la soumission au prince - en l'occurrence à son lieutenant le chevalier de Malte. Cette obéissance de principe et cette fidélité désintéressée leur ont coûté cher : les trois martyrs du Brésil sont moins, dans cette perspective, les martyrs de la Cause protestante que les martyrs de la fidélité opiniâtre à la monarchie.

En laïcisant le débat, jusqu'alors formulé en termes exclusivement religieux, en distinguant le point de vue politique de l'obéissance au souverain du point de vue de la conviction religieuse personnelle, en s'opposant sur ce point précis à son coreligionnaire Pierre Jurieu, qui inclinait de plus en plus à prôner la lutte armée contre Louis XIV, Pierre Bayle, quitte à récrire un peu l'histoire, engageait la réflexion dans une direction pleine d'avenir ${ }^{40}$.

En conclusion de cet aperçu sur les fortunes théologiques de la France Antarctique du Rio de Janeiro, on constate, chez la plupart des protagonistes du débat, une étrange indifférence aux enjeux de l'entreprise coloniale et missionnaire. Comme l'écrit Bruna Conconi au terme de son étude, le pressentiment d'avoir manqué une occasion importante, raté la rencontre avec l'autre et gâché une chance historique d'établir la concorde religieuse en terre étrangère ne semble pas même avoir effleuré la conscience de ces clercs et de ces intellectuels uniquement attachés à justifier et à théoriser leur engagement, à défendre aussi parfois leur existence ${ }^{41}$. 
Il est vrai que dans le temps où les controversistes s'agitaient ainsi autour du fantôme de la France Antarctique, les militaires quant à eux ne perdaient pas leur temps. En 1711, Duguay-Trouin s'emparait de Rio de Janeiro. À cette occasion des cartes topographiques furent levées par les ingénieurs de la flotte. L'île de Villegagnon, fortifiée de nouveau par les Portugais, figure en bonne place, à l'entrée de la baie et commandant la passe. Son nom est malmené. À côté de l'orthographe ancienne de "Villegaignon", qui est aujourd'hui encore celle de la nomenclature brésiliennne, on trouve la graphie déviante d"'Isle Gaignon", et dans les cartes portugaises de la même époque, celle de Vira-galhâo ${ }^{42}$, "Vire-le-Galion".

\section{De la mémoire à la fiction : fortunes actuelles de la France Antarctique}

Dans son essai de 1937, stimulant, mais inévitablement simplificateur, Afonso Arinos de Melo Franco soulignait à juste titre les liens souterrains qui unirent, à propos du Brésil, la Renaissance aux Lumières, et, dans le cas français, l'échec colonial de Villegagnon à la Révolution française. "Le type du 'bon sauvage', divulgué et célébré au XVIIIe siècle, fut inspiré par l'Indien américain", et plus spécialement brésilien, tel que le peignirent tour à tour le huguenot Jean de Léry, le sceptique Montaigne et les missionnaires capucins Claude d'Abbeville et Yves d'Évreux : vivant libre et nu, ignorant la propriété du sol, les procès et les querelles qui s'ensuivent ${ }^{43}$. Cette belle et grande fausse image, d'idée devenue dogme, eut un bel avenir, un avenir qui assiège notre présent et s'interpose entre la réalité historique et nous. À preuve les modernes fictions qui ont fleuri depuis vingt ans et qui ont pris la France Antarctique comme prétexte. Ce qui frappe, dans toutes ces adaptations inégalement soigneuses de l'exactitude anthropologique, c'est le prestige du mythe au détriment de l'histoire. La Renaissance a définitivement sombré avec sa complexité, ses contradictions, 
ses arguties scolastiques et sa grille théologique devenue aujourd'hui indéchiffrable. À la place, s'est levée une figure de substitution, plus séduisante, mais combien moins riche, celle de l'homme de la nature, laïcisée et retouchée par les Lumières ${ }^{44}$.

À l'automne 2001, l'extraordinaire succès rencontré par Rouge Brésil, le roman du médecin Jean-Christophe Rufin, Prix Goncourt de cette année-là, eut le mérite de porter l'aventure de la France Antarctique à la connaissance du grand public ${ }^{45}$. Un trio entrait dans la légende, composé d'un Thevet truculent et d'un Léry furtif, escortant un Villegagnon tonitruant et brouillon, débordant et colérique, le comble de la Renaissance à lui tout seul, rejetant dans l'ombre le hâbleur et le pasteur. Dans cette fiction de vaste ampleur, révélatrice des goûts de notre temps et aussi de ses malaises, un romancier au tempérament généreux traduisait dans l'histoire de deux enfants orphelins et exilés, trouvant parmi les Indiens du Brésil une nouvelle patrie, la nostalgie primitiviste qui hante notre société postmoderne.

Le plus réussi dans Rouge Brésil est le conte philosophique. Un conte dont les héros sont deux enfants, Just et Colombe, qui se croient frère et soeur. Il y a un parfum de Paul et Virginie, dans cette fiction transportée dans le Brésil du XVIe siècle, au temps de l'établissement colonial de Villegagnon. Le véritable lieu idéologique du livre de Jean-Christophe Rufin, ce n'est pas le Brésil de la Renaissance, mais les îles à sucre du siècle des Lumières. Le modèle est moins Léry ou Thevet que Bernardin de Saint-Pierre. Sa France Antarctique ressemble beaucoup au paradis de l'Ile de France, l'actuelle île Maurice, avec en prime le Pain de sucre et le Corcovado.

Just et Colombe, avant de se réunir pour une sorte de happy end en demi-teinte, symbolisent deux attitudes opposées, celle de la civilisation occidentale, lettrée, à prétention universelle; celle du monde indien, en symbiose avec la nature ${ }^{46}$. Colombe illustre la tentation naturiste, marquée par la tombée des vêtements, véritable lieu commun de la littérature sur le sujet, déjà présent dans le roman de Gilbert Pastor, Le Valet $d^{\prime}$ aventure $^{47}$. Le héros, Fabien Clément, échappé de Fort Coligny, vit nu à nue avec une Indienne pendant trois mois. Après avoir 
été fait prisonnier par des orpailleurs, puis par les Portugais vainqueurs des Français, qui lui font prêter serment de fidélité à leur roi, il disparaît dans la nature, un peu comme Just et Colombe à la fin de Rouge Brésil.

Plus lucidement, Jean-Christophe Rufin veut faire réfléchir sur la rencontre de deux humanités qui s'ignorent, comme le montre l'analogie entre les deux rites les plus spécifiques des deux cultures en présence, l'Eucharistie catholique et l'anthropophagie rituelle des Tupinamba ${ }^{48}$.

Le choix d'un couple d'enfants, avec ce que cela implique de légèreté, de fraîcheur et d'innocence, permet de déjouer le piège du voyeurisme et légitime en quelque sorte la tentation de lidylle. Colombe toute nue et peinte au génipat par les Indiennes qui l'accueillent comme une soeur, c'est une enfant rejoignant d'autres enfants. Le poncif du primitivisme retrouve de la sorte une nouvelle jeunesse, tout en entrant en résonance avec la pensée écologique de notre temps.

Le choix d'un couple d'enfants présente de surcroît l'avantage de prendre l'Histoire de biais, ou, si l'on préfère, à rebrousse-poil ; de maintenir vis-à-vis de l'emprise du passé, et d'un passé désagréable et tragique, la distance et l'ironie nécessaires. Du point de vue de la technique romanesque, il permet en outre à l'écrivain de conserver une marge de manoeuvre confortable, sans heurter la vraisemblance historique. Les prédécesseurs de Rufin étaient tombés dans cette erreur : en choisissant un personnage trop proche de Jean de Léry, qui reste le grand témoin sur la France Antarctique et un historien indépassable, Gilbert Pastor et Jean-Marie Touratier $^{49}$ avaient tenté l'impossible, à savoir réécrire ce chefd'oeuvre qu'est l'Histoire d'un voyage faict en la terre du Bresil. Leur échec, dès lors, était prévisible. Leurs romans sont de pâles copies, où la vigueur de l'original s'affadit de digressions et s'alourdit d'anachronismes.

Le premier, Le Valet d'aventure, présente en outre une vision presque constamment négative des Indiens, cruels, ivrognes et lubriques, ne trouvant de plus grand bonheur que dans de frénétiques orgies cannibales. Loin de cette 
consternante régression anthropologique qui puise dans les clichés les plus éculés sur le sauvage sans foi ni loi, et ramène loin en arrière de Léry ou même de Thevet, Jean-Marie Touratier fait preuve de plus d'élégance, mais la nervosité de sa narration, souvent très allusive, souffre de l'abus des phrases en langue indienne, si bien que certains chapitres de Bois rouge ressemblent à du "colloque tupi" délayé. Touratier a trop bien assimilé, semble-t-il, l'exemple du cosmographe André Thevet qui émaillait ses lourds in-folios de mots, d'expressions et de phrases entières tirés de vocabulaires français-brésilien à l'usage des négociants et des matelots.

Il est difficile d'imiter Jean de Léry. Plutôt que d'avoir en ligne de mire ce modèle, Rufin a pris ses distances par rapport à lui, la plus grande distance possible, en adoptant - nulle naïveté à cela, mais un très sûr métier - le point de vue censément vierge de deux enfants sans famille et sans héritage. De ce fait, l'intrigue ne croise qu'occasionnellement l'Histoire mère de Léry. Par une habileté suprême, Rufin fait comparaître furtivement Léry à la toute fin de son roman, personnage secondaire auquel le lecteur profane ne se doutera jamais que l'auteur doit l'essentiel de son information et surtout la qualité exceptionnelle d'un regard sur les réalités auparavant inouies du Nouveau Monde ${ }^{50}$.

Très habile et même ingénieux dans sa construction, Rufin ne l'est pas moins dans la recréation des personnages historiques, le cordelier et futur cosmographe du roi André Thevet, hâbleur et entiché de pratique, mais sachant à peine manier un astrolabe, rapatrié aussitôt que débarqué ; et surtout le tonitruant Villegagnon, "une sorte de Don Quichotte haut en couleurs et à l'humeur imprévisible" ${ }^{\prime 51}$. Seule minime erreur : Thevet est qualifié de "capucin"52, alors qu'il était tout simplement franciscain. Villegagnon est particulièrement réussi. Pas seulement parce qu'il acquiert des proportions gigantesques, qu'il fait transporter sur une frêle pirogue un lourd meuble Renaissance avec grotesques et cariatides ou qu'il aime l'art maniériste des disciples de Raphaël, faisant peindre une Madone à l'enfant dans la chapelle de Fort Coligny par un artiste 
tout exprès venu d'Italie. Pas seulement, non plus, en raison de ses colères homériques dont le souvenir nous a été transmis par Jean de Léry et les pamphlets protestants. Mais aussi et surtout parce que la psychologie et le comportement du personnage sont pleinement crédibles. Rufin a su faire son miel des analyses historiques récentes, et il en a tiré cette conclusion, développée dans la postface de son livre : "Loin d'être, comme le voulait la littérature de combat de chaque camp, un protestant renégat ou une victime des huguenots, Villegagnon devient ce "moyenneur" pour qui la Réforme est d'abord un idéal proche de l'humanisme, une tendance à retrouver la foi simple des origines" ${ }^{153}$.

La réhabilitation de Villegagnon a pour contrepartie la caricature de ses adversaires protestants - et c'est là sans doute le point faible du livre. Conte merveilleux des plus réussis, Rouge Brésil est plus contestable en tant que roman historique. J'écrivais dans l'avertissement de la troisième édition du Huguenot et le sauvage : "Contre l'austère et vétilleux Pierre Richer, représentant de Calvin et ministre de la Parole, le fulminant Villegagnon aura toujours le beau rôle" ${ }^{154}$. Dans son compte rendu publié dans Foi et Vie, Philippe de Robert va plus loin : "Même s'il est sous le charme, le lecteur protestant ne pourra sans doute pas s'affranchir d'un certain malaise devant les poncifs auxquels l'auteur a recours pour brosser ses personnages "genevois", toujours vêtus de noir, la mine grave, l'attitude austère, le comportement plein d'assurance et d'orgueil". C'est, conclut Philippe de Robert, "la caricature classique, dont l'uniformité contraste avec la diversité plus naturelle sous laquelle sont dépeints les personnages catholiques" ${ }^{155}$.

La charge, observe le même critique, culmine dans le personnage de la belle Aude, nièce du pasteur Richer - et création de l'auteur comme antithèse de Colombe - , farouchement attachée au triomphe de sa religion, manipulatrice et sournoise, à tel point que la tentative de meurtre imaginaire - qu'elle commet sur Just apparaît plus odieuse que les tortures et exécutions par noyade - bien réelles — infligées par Villegagnon à trois des colons protestants. On en vient à 
regretter que le romancier n'ait pas poussé jusqu'au registre tragique le portrait de cette Judith huguenote vindicative et passionnée.

Ce trait est d'autant plus regrettable que Rufin commet, ce faisant, une injustice flagrante à l'égard de sa principale source, qui est le huguenot Jean de Léry, cordonnier lors de l'expédition et plus tard pasteur. Il le dit lui-même dans sa postface :

[...] je tombai un jour, en rouvrant Léry, sur ces deux lignes : "Dans l'autre (navire) qui s'appeloit Rosée, du nom de celuy qui la conduisoit, en comprenant six jeunes garçons, que nous menasmes pour apprendre la langue des sauvages". Ces six enfants arrachés à leur orphelinat pour servir d'interprètes au milieu des tribus indiennes me firent quitter d'un coup l'espace aseptisé de l'Histoire, les abstractions de la politique ou de la religion [...]. Just et Colombe étaient nés et avec eux Rouge Brésil ${ }^{56}$.

Le roman serait donc né de deux lignes de Léry — ni de trois ni de quatre, mais de deux ! Mais pour mûrir et parvenir à son plein achèvement, il lui a fallu un peu plus. Ces deux enfants embarqués de force et bientôt isolés et fugitifs dans l'île du Brésil rappellent fort, par leurs émois adolescents et leur découverte du monde et d'eux-mêmes, Daphnis et Chloé, Paul et Virginie ou encore les jeunes héros de La Dispute de Marivaux. La situation est topique. Rufin, qui a beaucoup lu et beaucoup assimilé, écrit une variante de plus sur le mythe du couple primordial, à partir duquel le monde commence ou recommence. Cette dimension mythique est du reste à demi avouée, dans les toutes dernières lignes du roman:

Le plus attesté [des mythes Tupi] met en scène un déluge que le grand dieu Toupan aurait infligé aux hommes qui l'avaient courroucé. Toute l'humanité y périt, à l'exception d'un frère et d'une soeur. De leur union devait naître la nouvelle race humaine. [...] Personne ne pourra nous empêcher de voir, derrière ces deux héros du mythe, la trace de deux personnages que nous avons aimés, tout ce qui reste de Just et de Colombe ${ }^{57}$. 
Dans le processus de création romanesque, c'est, bien sûr, le contraire qui est vrai. Le mythe des deux Jumeaux, dans la cosmogonie tupinamba, n'est pas le vestige du roman; c'est le roman, à l'inverse, qui découle du mythe des Jumeaux, ou plutôt de la rencontre, par-delà les mers, d'un mythe indien et de mythes littéraires européens, dont il additionne et combine les variantes.

S'il n'est pas tout à fait exact que Rouge Brésil ait surgi, tout désarmé, dans la fraîcheur et l'innocence retrouvées, de deux lignes de Léry, il reste que le roman emprunte beaucoup à ce dernier : atmosphère, personnages taillés à la serpe, détails ethnographiques, realia exotiques, couleurs et scènes, tableaux et parures. Pour le dire en un mot, Rufin doit à Léry quelque chose de cette présence extraordinaire et presque hallucinatoire qui émane de l'Histoire d'un voyage et qui transporte jusqu'à nous, par un charme puissant, les lointains dans l'espace et dans le temps.

Or l'ingratitude de Rufin tant à l'encontre de Léry que des protestants est patente. Les voici chassés d'une histoire qu'ils nous ont transmise et qui est au départ la leur, expulsés d'une vision du monde dont nous leur sommes entièrement - et presque exclusivement - redevables. Nous, c'est-à-dire JeanChristophe Rufin et son demi-million de lecteurs.

La caricature des protestants, observe Philippe de Robert, "semble devenue maintenant un fait culturel qu'il est difficile de modifier - autant dire qu'il faut vivre avec ${ }^{1158}$. De toute évidence, pour l'opinion commune aujourd'hui, les réformés du XVIe siècle représentent une étrangeté plus radicale que celle des Tupinamba anthropophages. Le fait est qu'ils semblent vouloir concilier l'inconciliable. En matière de controverse eucharistique, leur rationalisme peut séduire, mais leur opiniâtreté décourage ou révolte. On est prêt à s'apitoyer sur leur sort de victimes, mais ces victimes du fanatisme se montrent à leur tour plus fanatiques que leurs persécuteurs. C'est déjà ce que pensait Voltaire des camisards révoltés des Cévennes, assez fous pour tenir tête aux troupes du Roi-Soleil, parce que le Saint-Esprit leur parlait. Leurs souffrances non- 
pareilles méritent qu'on les plaigne, mais leur orgueil doit être blâmé.

Toute polémique mise à part, et tout en regrettant que l'auteur soit tombé dans le poncif antiprotestant, on peut aussi voir dans cette charge anti-huguenote un réflexe bien compréhensible, le réflexe de défense, et donc d'ingratitude, du romancier vis-à-vis de l'historien. Je ne parle pas ici de déontologie, Jean-Christophe Rufin étant à cet égard au-dessus de tout soupçon - à la différence notoire de quelques-uns de ses suiveurs, les plus médiocres s'entend. On ne peut qu'être frappé, au contraire, de l'exactitude scrupuleuse avec laquelle, dans sa postface "À propos des sources de Rouge Brésil", il reconnaît ses dettes. Je veux ici parler d'un phénomène plus profond et plus secret, d'une sorte de réaction instinctive et en général inavouée.

Car dans l'ingratitude de Rufin pour le "corpus huguenot" en général et pour Léry en particulier, il y a tout autre chose que l'antiprotestantisme vulgaire : le ressentiment naturel, ou, pour mieux dire, organique, de l'auteur de fiction pour la source qu'il phagocyte et qu'il doit, autant que possible, faire disparaître pour se l'incorporer et la transformer dans sa propre substance. "Frustration et paralysie", constate lucidement Rufin, tels sont les sentiments que la masse des documents et des interprétations oppose au processus créateur. Force est au romancier de faire le vide, ou du moins de feindre de dédaigner, voire d'oublier, ce qui, par nécessité, constitue le matériau qu'il va mettre en oeuvre. "Ce qui pour l'historien est une fin - décrire des faits - n'est pour le romancier qu'un début : il doit passer du thème à l'intrigue, des événements d'ensemble aux actions particulières. Pour cela, il lui faut de l'air, de l'espace, bref, de l'inconnu. Et surtout de l'émotion"59.

De la mémoire historienne il faut donc faire table rase, pour laisser libre cours à l'imagination "et surtout à l'émotion". Quitte à revenir ensuite à l'Histoire, mais par le détour de la rêverie, de la libre association d'idées et de l'incorporation. C'est ainsi qu'une courte période de jeûne facilite la digestion. Le docteur Rufin sait de quoi il parle, et la métaphore alimentaire est parfaitement 
à sa place dans ce roman qui parle d'authentiques cannibales. "Il faudrait toujours s'imposer cette digestion, à la suite de laquelle on voit plus clair" ${ }^{110}$. Une digestion réussie, c'est, de la part du romancier, une amnésie partielle et contrôlée. À quoi s'ajoute un soupçon d'anachronisme, pour assaisonner l'histoire ancienne au goût de notre temps. C'est cette tâche tout à la fois digestive et culinaire que Jean-Christophe Rufin a accomplie avec succès, et dont le public, massivement, lui a su gré.

Bien entendu, l'analyse qui précède appelle des nuances: la tendance à caricaturer la Réforme est contrebalancée, dans Rouge Brésil, par le fait que la vision des événements est une vision réformée. Réformé, le portrait de Villegagnon colérique et magnifique, avec les correctifs imposés par l'historiographie récente. Réformée la critique de l'intolérance de l'Église catholique - mais l'auteur n'a pu s'empêcher de rappeler, par le truchement du dissident Quintin, la malheureuse affaire Servet, stigmatisant au passage "l'horrible Théodore de Bèze", qui a justifié dans une brochure le brûlement du médecin espagnol ${ }^{61}$. Réformées encore, l'histoire de la colonie et l'explication de son échec. Réformée même, la caricature des anabaptistes et des libertins spirituels, présentés comme de dangereux extrémistes, des "enragés" capables des pires atrocités : ne mettent-ils pas en pièces, avant de les exposer, comme sur un étal de boucherie, le corps de leurs adversaires ? Réformé, bien sûr, le récit de la controverse eucharistique ${ }^{62}$, avec toutefois l'ajout de quelques erreurs tenant à la méconnaissance du dogme. Contrairement à ce qui est suggéré, la Cène calviniste est rare. À la différence de l'Eucharistie catholique, elle ne sustente pas. C'est pourquoi elle ne constitue pas un besoin aussi fort, aussi physique, aussi vital, que pour un catholique la Messe. - Mais reprochera-t-on à un romancier de n'être pas théologien?

Toujours est-il qu'en défaisant le nœud inextricable entre le désir d'ailleurs et la passion protestante, qui déboucha par un beau printemps 1557 sur le rêve d'une institution chrétienne en terre païenne, dans un monde presque neuf, loin de la corruption de la vieille Europe, Jean-Christophe Rufin a tout à la fois brouillé l'Histoire et simplifié l'événement. Il a brouillé l'Histoire, 
puisque le XVIe siècle, dans ses contradictions et ses rêves, est ici à peine reconnaissable. Jugé brutal et compliqué, insupportable de fanatisme, le voici donc filtré par l'idéologie progressiste des Lumières, repeint aux couleurs pastel du style rococo et rhabillé (ou plutôt déshabillé) à la mode du Bon Sauvage. Rufin a d'autre part simplifié l'événement, en le vidant de sa substance symbolique, pour le remplir d'un imaginaire de substitution, en définitive assez banal. De sorte que si, grâce à Rouge Brésil, l'épisode de la France Antarctique est enfin lisible et connu de tout un chacun, c'est au prix d'une transparence illusoire et d'un réel appauvrissement. Mais pouvait-il en être autrement?

LESTRINGANT, Frank. The Memory of the French Antarctic. História, v.27, n.1, p.101- 134, 2008.

\begin{abstract}
The wealth of information about the French Antarctic is incommensurable due to its short experience in colonization in Brazil from November 1555 to March 1560. It was restricted to a small geographical space near the cost of the bay of Rio de Janeiro. I will start in this text from the judgment of Abade Prévost, author of the immortal work Manon Lescaut, as well as his Histoire Générale des Voyages to reconstitute the memory of the French Antarctic in French Historiography.
\end{abstract}

Keywords: French Antarctic; Memory; Prévost.

\title{
NOTAS
}

${ }^{1}$ Abbé Prévost, Histoire generale des voyages, t. XIV, 1757, chap. IX, p. 309, note 18. Citant Jean de Léry, Histoire d'un voyage faict en la terre du Bresil, Paris, LGF, "Bibliothèque classique", 1994, chap. XIII, p. 310 313.

2 Abbé G.T.F. Raynal, Histoire philosophique et politique des établissements et du commerce des Européens dans les deux Indes, Amsterdam, 1770, t. III, livre IX, chap. VII, p. 541-542. — Pour le 
commentaire de cette filiation de Prévost à Raynal, voir Frank Lestringant, Le Huguenot et le sauvage. L'Amérique et la controverse coloniale, en France, au temps des guerres de Religion, 3e éd., Genève, Droz, 2004, p. 202.

3 Afonso Arinos de Melo Franco, O Indio brasileiro e a Revoluçâo francesa, Rio de Janeiro, José Olimpio, 1937. Cet ouvrage vient tout juste d'être traduit en français sous le titre : L'Indien brésilien et la Révolution française, trad. par Monique Le Moing, Paris, La Table Ronde, 2005.

${ }^{4}$ Jean de Léry, Histoire d'un voyage, op. cit., chap. XXI, p. 508.

${ }^{5}$ Ibid., p. 508.

${ }^{6}$ Montaigne, Essais, I, 31, éd. Pierre Villey, Paris, PUF, 1965, p. 205.

${ }^{7}$ David El-Kenz, Les bûchers du roi. La culture protestante des martyrs (1523-1572), Seyssel, Champ Vallon, 1997.

8 Thierry Wanegffelen, Ni Rome ni Genève. Des fidèles entre deux chaires en France au XVIe siècle, Paris, Champion, 1997.

${ }^{9}$ Arthur Heulhard, Villegagnon, roi d'Amérique. Un homme de mer au XVIe siècle (1510-1572), Paris, Ernest Leroux, 1897. Ce livre bien informé en son temps, mais très tendancieux du point de vue de l'interprétation historique, n'a pas été remplacé depuis. Le livre très fautif de Léonce Peillard, Villegagnon, Vice-Amiral de Bretagne, ViceRoi du Brésil, préface d'Alain Peyrefitte, Paris, Perrin, 1992, et celui de Lucien Provençal et Vasco Mariz, Villegagnon. Un chevalier de Malte au Brésil, Paris, Éditions Rive droite, 2001, n'ont ni l'un ni l'autre la prétention de se substituer à cette somme.

${ }^{10} \mathrm{Ce}$ que fait, en un sens large et légèrement abusif, Serge Elmalan dans son livre, Villegagnon ou A Utopia tropical. Romance, traduçâo de Procopio Abreu, Rio de Janeiro-Sâo Paulo, Editora Record, 2004 (traduction de : Nicolas de Villegagnon ou l'utopie tropicale, Lausanne, Éditions Favre, 2002). L'auteur a, bien sûr, l'excuse de la fiction romanesque.

${ }_{11}$ J'emprunte cette formule à Olivier Christin, La Paix de Religion. L'autonomisation de la raison politique au XVIe siècle, Paris, Éditions du Seuil, 1997.

12 Je résume ici un propos développé dans mon livre : Une sainte horreur ou le voyage en Eucharistie (XVIe-XVIIIe siècle), Paris, P.U.F., 1996, p. 85-118.

${ }^{13}$ Pour reprendre le mot d'Henri Estienne, Introduction au Traité de la conformité des merveilles anciennes avec les modernes, ou Apologie pour Hérodote, Genève, 1566, "Au lecteur", f. â 6 r $^{\circ}$. 
${ }^{14}$ Cité par Bernard Cottret, 1598. L'Édit de Nantes. Pour en finir avec les guerres de religion, Paris, Perrin, 1997, p. 164.

${ }_{15}$ Ce concept de "révolution sociologique" est dû à Roger Caillois, préface à Montesquieu, Euvres complètes, Paris, Gallimard, "Bibliothèque de la Pléiade", 1947, t. I, p. V.

${ }^{16}$ Plaute, Asinaria, v. 495. Cf. Érasme, Adages, I, I, 70.

${ }^{17}$ Jean de Léry, op. cit., chap. XIV, p. 339.

18 Plaintes des Eglises Reformées de France, sur les violences et injustices qui leur sont faites en plusieurs endrois du Royaume, et pour lesquelles, elles se sont en toute humilité et diverses fois adressées à sa Majesté, M.D.XCVII., p. 109.

${ }^{19}$ Ibid., p. 106-107.

${ }^{20}$ Pour ce concept d'anthropémie, voir Claude Lévi-Strauss, Tristes Tropiques, Paris, Plon, 1955, chap. XXXVIII, p. 447-448.

${ }^{21}$ Plaintes des Eglises, ibid., p. 110.

${ }^{22}$ Voir sur ce point Max Engammare, "L'inhumation de Calvin et des pasteurs genevois de 1540 à 1620 . Un dépouillement très prophétique et une pompe funèbre protestante qui se met en place", Les Funérailles à la Renaissance, éd. par Jean Balsamo, Genève, Droz, 2002, p. 271-293.

${ }^{23}$ Bernard Cottret, loc. cit.

${ }^{24}$ Voir Une sainte horreur ou le voyage en Eucharistie, op. cit., passim.

${ }^{25}$ Voir sur ce point F. Lestringant, L'Expérience huguenote au Nouveau Monde (XVIe siècle), Genève, Droz, 1996, p. 141-153.

${ }^{26}$ Théodore de Bèze, Les Vrais Pourtraits des hommes illustres en pieté et doctrine, du travail desquels Dieu s'est servi en ces derniers temps, pour remettre sus la vraye Religion en divers pays de la Chrestienté, Genève, Jean de Laon, 1581 (réimpr. Genève, Slatkine Reprints, 1986), p. 177-179. L'édition latine originale est de 1580.

${ }^{27}$ Lyon, Simon Rigaud, 1622. Sur l'Antimartyrologe de Jacques Severt, voir Arthur Piaget et Gabrielle Berthoud, Notes sur le Livre des Martyrs, Neuchâtel, Université de Neuchâtel, 1930, chap. VII, p. 100-135.

${ }^{28}$ Voir sur ce point Frank Lestringant, La Cause des martyrs dans les Tragiques d'Agrippa d'Aubigné, Mont-de-Marsan, Éditions InterUniversitaires, 1991 ; Lumière des martyrs. Essai sur le martyre au siècle des Réformes, Paris, Champion, 2004, 1ère partie: "La Cause des martyrs", p. 19-110.

29 Florimond de Raemond, L'Histoire de la naissance, progrez et decadence de l'heresie de ce siecle, Paris, veuve Guillaume de La Noue, 1610, VII, 6, p. 866 : "Les Hérétiques peuvent pâtir pour Jésus-Christ sans être martyrs". 
${ }^{30}$ J. Severt, op. cit., VII, II, p. 595.

${ }^{31} \mathrm{~J}$. Severt, ibid.

${ }^{32}$ Le mot est attribué à Pierre Richer par Jean de Léry, op. cit., chap. VI, p. 165.

${ }^{33}$ Sur cette méprise possible et le malentendu qui en résulta, voir Thierry Wanegffelen, "Rio ou la vraie Réforme: la 'France Antarctique' de Nicolas Durand de Villegagnon entre Genève et Rome", in Naissance du Brésil moderne, 1500-1808, sous la direction de Katia de Queiros Mattoso, Idelette Muzart-Fonseca Dos Santos et Denis Rolland, Paris, Presses de l'Université de Paris-Sorbonne, 1998, p. 159-175.

34 J. de Léry, op. cit., chap. XXII, p. 549. Cf. Silvia Shannon, "Villegagnon, Polyphemus, and Cain of America: Religion and Polemics in the French New World", in Michael Wolfe ed., Changing Identities in Early Modern France, Durham, NC, Duke University Press, 1996.

${ }^{35}$ J. Severt, op. cit., p. 597.

${ }^{36}$ Ibid., p. 598.

${ }^{37}$ Ibid.

38 Pierre Bayle, Dictionnaire historique et critique, seconde édition, Rotterdam, Reinier Leers, 1702, où trois articles sont consacrés à la France Antarctique du XVIe siècle, s. v. "Leri", "Richer" et "Villegaignon".

39 Ronsard, Continuation du Discours des Miseres de ce temps, à la Royne (1562), v. 120-122, in Euvres complètes, éd. Paul Laumonier, t. XI, Paris, Société des Textes Français Modernes, 1973, p. 42. Cf. Une sainte horreur, op. cit., p. 151-152.

${ }^{40} \mathrm{~J}$. de Léry, Histoire d'un voyage, op. cit., chap. VI, p. 192-195 : " nous avions bien moyen de l'en chasser lui-même [i.e. de l'île Coligny] si nous eussions voulu $"$.

41 Je résume ici la contribution de Bruna Conconi, "Les Fortunes de la France Antarctique de Villegagnon au temps de la crise de la conscience européenne: les métamorphoses d'une controverse", in La France-Amérique (XVIe-XVIIIe siècles). Actes du XXXVe Colloque international d'études humanistes, réunis par Frank Lestringant, Paris, Champion, 1998, p. 143-166.

${ }^{42}$ Ibid., p. 166.

${ }^{43}$ Voir par exemple Joâo Teyxeira, Descripçâo de todo o maritimo da terra de Santa Crus chamado vulgarmente o Brazil. Anno 1690, pl. 9, f. 18.

${ }^{44}$ Afonso Arinos de Melo Franco, op. cit., 2005, p. 228.

${ }^{45}$ Sur l'écran que forme entre le XVIe siècle et notre époque le siècle des Lumières, voir l'argumentation de Laurent Loty, "Anthropologie, 
religion et politique : Images du XVIe siècle au XVIIIe siècle... selon le XXe siècle", Cahiers d'histoire culturelle (Université de Tours, UFR de Lettres), $\mathrm{n}^{\circ} 11$ : "Les Représentations du XVIe siècle et de la Renaissance aux XVIIIe et XIXe siècles", 2002, p. 245-259.

${ }^{46}$ Jean-Christophe Rufin, Rouge Brésil, Paris, Gallimard, 2001.

${ }^{47}$ Philippe de Robert, "Protestantisme et saga brésilienne", Foi et Vie, février 2002, p. 69-71, compte rendu de Jean-Christophe Rufin, op. cit.

${ }^{48}$ Gilbert Pastor, Le Valet d'aventure, Paris, Balland, 1990.

${ }^{49}$ Philippe de Robert, art. cit., p. 70.

${ }^{50}$ Gilbert Pastor, Le Valet d'aventure, op. cit. ; Jean-Marie Touratier, Bois rouge, Paris, Galilée, 1993.

${ }^{51}$ J.-Ch. Rufin, Rouge Brésil, p. 594.

${ }^{52}$ Philippe de Robert, art. cit., p. 70.

53 J.-Ch. Rufin, Rouge Brésil, p. 187.

${ }^{54}$ Ibid., p. 600-601.

55 F. Lestringant, Le Huguenot et le sauvage, 3e édition revue et augmentée, Genève, Droz, 2004, p. 12.

${ }^{56}$ Philippe de Robert, art. cit., p. 70.

${ }^{57}$ J.-Ch. Rufin, Rouge Brésil, p. 601-602.

${ }^{58}$ Ibid., p. 595.

${ }^{59}$ Philippe de Robert, art. cit., p. 71.

${ }^{60}$ J.-Ch. Rufin, Rouge Brésil, p. 601.

${ }^{61}$ Ibid., ad loc.

62 Ibid., p. 362-363. D'après Arthur Heulhard, op. cit., p. 174.

63 J.-Ch. Rufin, Rouge Brésil, p. 443-446

Artigo recebido em 03/2008. Aprovado em 07/2008. 
(20) 